\title{
COSMIC BACKGROUND RADIATION LIMITS FOR SETI
}

\author{
S. Gulkis \\ Jet Propulsion Laboratory, California Institute of Technology, Pasadena, CA 91109 USA
}

\section{$\underline{\text { Summary }}$}

Searches for extraterrestrial intelligence (SETI) depend ultimately on the noise introduced in the receiving system by the photon flux from cosmic background radiation. The major sources of the diffuse background radiation include galactic radiation at decimeter wavelengths and longer, $2.7 \mathrm{~K}$ radiation at millimeter wavelengths, interstellar dust at submillimeter wavelengths, and interplanetary dust and stellar emission at infrared wavelengths. Our knowledge of these has greatly improved due to recent measurements from the IRAS satellite. We give estimates for various background radiation components over wavelengths from 1 micrometer to 10 meters. Signal-to-noise estimates are presented for background limited linear and direct photon detection systems. Optimal wavelength regions for SETI are discussed. The discussion in this presentation follows a similar discussion given by C.H. Townes (Proc. Natl. Acad. Sci. USA, Vol. 80, pp. 1147-1151, February 1983).

\section{Discussion}

\section{N. EVANS: Could you define more precisely what you mean by the microwave window?}

S. GULKIS: The sum of three noise sources - 1) galactic synchrotron emission, 2) cosmic microwave background radiation, and 3 ) the spontaneous emission associated with coherent receivers - defines a broad spectral region from about $1 \mathrm{GHz}$ to $60 \mathrm{GHz}$ where background noise emission is at a minimum. This spectral region is known as the free-space microwave window. From the surface of the earth, the window is somewhat narrower due to the emission from the terrestrial atmosphere. The resulting minimum brightness region including the terrestrial atmosphere is known as the terrestrial microwave window. It extends from approximately $1 \mathrm{GHz}$ to $10 \mathrm{GHz}$. 